\title{
Predicciones del fin del mundo: Astronomía y ciencia ficción en los magazines de editorial Zig-Zag $(1900-1920)^{*}$
}

\author{
Verónica Ramírez
}

\section{Resumen}

Durante las primeras décadas del siglo XX, gracias a la modernización de la industria editorial, las predicciones del fin del mundo asociadas a daños ocasionados por fenómenos celestes circularon como leitmotiv de folletines y de textos de divulgación, produciéndose una expansión de la ciencia ficción más allá de los límites literarios. Posibles choques de astros con la Tierra y teorías científicas acerca de la toxicidad de la cola de los cometas, provocaron que dentro de los magazines cercanos al paso del cometa Halley de mayo de 1910, proliferaran obras de ciencia ficción, así como textos divulgativos que imaginaban posibles situaciones apocalípticas ocasionadas por este fenómeno celeste. En ese sentido, nuestro trabajo analiza la utilización del saber astronómico para propósitos literarios, así como el uso del tono literario para difundir y popularizar la ciencia en los magazines de la editorial Zig-Zag.

Palabras clave: Astronomía, ciencia ficción, popularización de la ciencia, predicciones, magazines.

\section{Predictions of the End of the World: Astronomy and Science fiction in Zig-Zag Magazines (1900-1920)}

\begin{abstract}
The predictions of the end of the world associated with damages caused by celestial phenomena have been in our history for a long time. During the first decades of the 2oth Century, thanks to the modernization of the publishing industry, this theme circulated in magazines as a leitmotiv of literature and dissemination texts, producing an expansion of science fiction beyond literary limits. Possible collisions of stars with the Earth and scientific theories about the toxicity of the tail of comets, caused that in the editions of the magazines near the Comet Halley passage in May 1910, has proliferated science fiction works, as well as informative texts that imagined possible apocalyptic situations caused by this celestial phenomenon. In this sense, our work analyzes the use of astronomical knowledge for literary purposes, as well as the use of the literary tone to disseminate and popularize science in magazines of the Zig-Zag publishing house.
\end{abstract}

Este trabajo fue desarrollado en el marco del proyecto Fondecyt Posdoctoral №3180131, titulado “Astronomía y literatura en Chile: diálogo y discusión en el espacio público (1880-1930)", actualmente en ejecución.

** Chilena. Doctora en Literatura Chilena e Hispanoamericana por la Universidad de Chile. Académica e investigadora de la Universidad Adolfo Ibáñez, Santiago, Chile.vramirez@uai.cl 
Keywords: Astronomy, Science fiction, Popularization of science, Predictions, Magazines.

\section{Introducción}

La literatura occidental posee una larga tradición asociada a las predicciones y representaciones del fin o destrucción del mundo. La poesía antigua, por ejemplo, era vista como una práctica asociada a propiedades esotéricas, en la que el poeta tenía más características comunes con un mago que con un artista de la palabra, como puede verse en las obras de Homero, idea que a menudo le atribuyó cualidades predictivas a la creación poética (Latorre, "La poesía de la muerte..." 4). Por otra parte, "el tema del fin del mundo ha sido, desde sus mismos inicios, una constante en el pensamiento y la literatura hispanoamericanos" (Castany, "El motivo del fin del mundo..." 183), tradición que se ha sostenido a través de las religiones en general, y el cristianismo en particular, como explica el mismo autor (185). El milenarismo, así como el apocalipticismo, mesianismo y escatologismo, se han visto ligados a bases doctrinales oscuras y contradictorias, de tal modo que "numerosos teólogos, profetas e iluminados tratarán de determinar el momento y las características del fin de los tiempos" (186). Aunque el milenarismo desde los últimos siglos continúe existiendo de forma marginal, la temática se ha fundado en otro tipo de bases, tales como libros apócrifos, visiones o revelaciones orales, así como predicciones astronómicas (186).

En relación con esto último, la lista de casos donde los fenómenos astronómicos han sido asociados con el fin del mundo es igualmente extensa a lo largo de nuestra historia. Si solo nos centramos en el siglo XIX, encontramos entre otras, la predicción de 1852 de Temístocles Zena, director del Observatorio de Palermo, así como un anuncio predictivo fechado para el 13 de junio de 1857, o las predicciones de 1872, 1881 y 1885.

Quizás algunos de los pronósticos más recordados de ese siglo sean los del astrónomo austriaco Rodolfo Falb y del inglés Thomas Harris, quienes de manera independiente convinieron que la vida en la Tierra sucumbiría producto de fenómenos celestes el 13 de noviembre de 1899. Harris defendía que en esa fecha la Tierra sería atravesada por un grupo de asteroides conocidos como las estrellas errantes Leónidas, 
pertenecientes a la constelación de León. Pero lo cierto es que todos los años en noviembre nuestro planeta atraviesa dicha agrupación de asteroides inofensivos, y cada treinta y tres, la coincidencia llega a su máxima expresión, situación que justamente se vivió en 1899, favoreciendo en cierta medida la teoría de Harris. El astrónomo austriaco, por otra parte, relacionaba la destrucción del planeta con el paso del cometa Biela, el que supuestamente chocaría de manera irremediable con el globo terráqueo, haciéndolo explotar.

Si bien — como es evidente—, ninguna de estas predicciones se concretó, la prensa nos demuestra que la población siguió interesándose en este tipo de anuncios. En Chile, el terremoto de 1906, con epicentro en Valparaíso, generó un ambiente de conmoción en la sociedad que sugestionó a las personas a creer en fechas fatídicas ${ }^{1}$. Este ambiente apocalíptico volvió a encenderse durante los años y meses previos al paso del cometa Halley de 1910, situación que no solo se vivió en nuestro país, sino también en distintos lugares del mundo, como podemos enterarnos a través de la prensa ${ }^{2}$.

La prensa funcionaba como un dispositivo a través del cual circulaban estas teorías predictivas asociadas al saber astronómico. En las páginas de diarios, periódicos y revistas, podemos enterarnos del trato que los científicos y los públicos de la ciencia brindaron a los anuncios acerca de la destrucción del mundo asociada a fenómenos celestes, así como conocer la manera en que circuló y se resignificó ese conocimiento en contextos globales y locales. Debemos destacar que la prensa adquirió una importancia fundamental en los procesos de circulación del conocimiento científico desde el siglo XIX en adelante ${ }^{3}$.

En este trabajo se estudia cómo se nutre la creación literaria a partir de la circulación de teorías astronómicas predictivas y cómo inciden las obras literarias en la generación y circulación de nuevo conocimiento astronómico asociado a anuncios del fin del mundo. Es decir, analizaremos

En esos años los sismógrafos en nuestro país eran operados dentro de los observatorios astronómicos, puesto que no existía una institución específica que se hiciera cargo de esta materia. La sismología, a su vez, recién comenzó a concebirse como una disciplina específica en esas mismas décadas (Valderrama, "La catástrofe" 177). A esto debe sumarse que entre las teorías respecto de la causa de los terremotos más populares, la influencia de los astros sobre la Tierra era una de ellas, donde Falb figura como uno de sus defensores (Valderrama, Observando 1).

2 Pedro Ruiz-Castell (2011), por ejemplo, estudió el carácter letal que se le atribuyó al paso del Cometa Halley en el contexto español ("Priority Claims and Public Disputes...” 174).

3 Para profundizar en este tema, véase Geoffrey Cantor et. al. (2004). 
cómo se relacionan el saber astronómico y el literario —en específico, el género de ciencia ficción- en situaciones en las que se ha pronosticado el fin del mundo. La literatura, como ha señalado Agustí Nieto-Galán (2011), ha encontrado a lo largo de la historia, múltiples puntos en común con la ciencia (Los públicos 73-80), relación que se enmarca dentro de la interacción que sostienen los científicos y los públicos de la ciencia, la que a su vez permite el intercambio o transferencia entre distintos saberes. Asimismo, como ha explicado Lorena Valderrama (2016), terremotos, cometas y eclipses ofrecen atractivos espectáculos para científicos y audiencias más amplias, convirtiendo estos fenómenos en experiencias sublimes y elevando la imaginación hasta el punto de lo no imaginable, donde las palabras pueden llegar a ser impotentes para representar lo vivido ("La catástrofe..." 169 y 174), o bien aquellas —las palabraspueden recurrir a la ficción.

En este sentido, una fuente interesante para constatar esta relación entre literatura y astronomía en el periodo estudiado es la revista cultural y, en forma específica, los magazines que comienzan a circular a principios del siglo XX. Primero, porque como han definido Ossandón y Santa Cruz (2005), los magazines gozan de una naturaleza híbrida y miscelánea, "capaz de albergar en su interior en forma entremezclada crónicas, entrevistas, reportajes de actualidad, ilustraciones, avisos publicitarios, cuentos y novelas por entrega, notas de vida social, caricaturas, poemas, etc." (33), característica que favorece el diálogo entre distintas disciplinas y saberes — tales como la literatura y la ciencia- de manera más intensa que en el diario noticioso o en la prensa diaria. Por otra parte, los magazines incluyeron ilustraciones y fotografías de manera abundante, lo que permitió complementar con imágenes la escritura de ficción que surgió a partir de estos fenómenos celestes. Se debe considerar también que las empresas que produjeron magazines a principios del siglo XX introdujeron maquinaria de impresión moderna, así como nuevos soportes materiales, acelerando el ritmo de impresión, tiraje, e incluyendo iconografía, lo que hizo a estas revistas más atractivas y accesibles al público menos letrado (Alvarado 126-127), y generando mayor amplitud en sus audiencias. Los magazines, por lo tanto, eran dispositivos que favorecían el diálogo interdisciplinario transmitiendo diversas temáticas a audiencias de estratos sociales amplios, heterogéneos y diversos, teniendo cabida en ellos, tanto públicos expertos, como legos en distintas materias. 
En Chile, la editorial Zig-Zag fundada por Agustín Edwards MacClure y Gustavo Helfmann en 1905, lideró la producción y distribución de revistas tipo magazines durante los primeros años del siglo XX. Antes de asociarse estos dos empresarios, Helfmann había creado la revista ilustrada Sucesos que circuló entre 1902 y 1932, y Edwards había fundado Revista Zig-Zag (principios de 1905), cuyo último número se publicó en 1964. Bajo el alero de esta empresa se editaron múltiples revistas, destacando por su contundente y extenso alcance las dos ya mencionadas (Sucesos y Zig-Zag) y los magazines Corre y vuela (19081927) y Pacífico magazine (1913-1921)4. Nuestro estudio se centra en las ediciones publicadas en las dos primeras décadas del siglo XX de estas cuatro revistas de la casa Zig-Zag. Si bien todas ellas tienen en común la intención de abarcar un público amplio, Corre y vuela se dirigió hacia un público más popular; Pacífico Magazine, a un grupo más selecto, culto y exigente; mientras que Sucesos y Revista Zig-Zag fueron más transversales, enfocadas en los intereses de la clase media. Por consiguiente, la editorial contaba con una variada gama de lectores y consumidores de distintos niveles socioculturales.

La justificación del periodo de este análisis responde, en primer lugar, a esa etapa del desarrollo de la cultura impresa en nuestro país antes descrita y al tratamiento de la ciencia como un negocio o mercancía que se compra y se vende - gracias a la expansión y desarrollo de la divulgación impresa- (Nieto-Galán, "La ciencia en la esfera..." 66); y, en segundo lugar, a la concentración en esos años de la circulación de teorías predictivas asociadas a fenómenos celestes.

Nuestra aproximación teórica hacia el saber científico responde a perspectivas que han marcado los estudios históricos y sociológicos de la ciencia de las últimas décadas, los que han tendido a considerarla como un proceso y no como un producto acabado, en el cual participan distintos grupos de la sociedad (Shapin \& Schaffer, 1985; Latour, 1992). Esto último nos conduce a comprender el desarrollo de la ciencia vinculado a "la construcción de espacios y prácticas de sociabilidad" (Sanhueza 15). Esta perspectiva, a su vez, entiende la ciencia en sí

Otros magazines de esta empresa fueron El Peneca (1908-1960), Familia (1910-1928 y 1935-1940) y Selecta (1909-1912). La primera revista estaba dirigida a una audiencia infantil; la segunda al público femenino, y la tercera fue destinada al fomento exclusivo del arte y la literatura. En este trabajo no consideraremos estas tres revistas, ya que nos enfocaremos en aquellas que mantuvieron como esencia la multiplicidad temática y que apuntaron hacia una audiencia más heterogénea. 
misma como un proceso comunicativo, donde es imposible separar su práctica de su comunicación, de tal modo que todo acto científico es, a su vez, un acto comunicativo (Secord, "Knowledge in transit" 654). Ello se justifica, en parte, en que el conocimiento es palpable en la medida en que se transmite, pero sobre todo, en que la práctica científica no puede entenderse como un ejercicio apartado y distanciado de la sociedad, sino como uno que es efectuado dentro y por la sociedad, lo que implica "entender a las audiencias como activas y prestar más atención a las prácticas de lectura, circulación, traducción, distribución, apropiación y otros procesos de comunicación científica, prestando atención a la transformación y resignificación de los conocimientos" (Valderrama, Observando 26). En ese sentido, todos somos posibles agentes de producción de conocimiento científico (Broks, "Science, Media and..." 124), lo que significa que las revistas culturales pueden ser consideradas soportes no solo de circulación, sino también de generación de saberes, y en el caso puntual de nuestro análisis, de conocimiento astronómico.

Por consiguiente, este trabajo se articula en función de las siguientes preguntas: ¿Cómo interactúan el saber astronómico y el saber literario en magazines chilenos a principios del siglo XX? ¿En qué medida las predicciones del fin del mundo incentivan y promueven la relación entre el campo astronómico y el literario en este periodo? ¿Cómo inciden las teorías astronómicas en la creación literaria y cómo influyen las obras literarias en la generación de conocimiento astronómico?

\section{La astronomía en magazines chilenos}

Durante los últimos años del siglo XIX y los primeros del XX destacó el discurso modernizador del Centenario de nuestra República, en el que nuestro país, al igual que otras naciones latinoamericanas, se mostraba deseoso de ser visto como uno de características prósperas. En dicho contexto finisecular se concretaron importantes avances materiales en diversas áreas, tales como salud e higiene, viabilidad, transporte, comunicación y educación, entre otros. La modernización también se manifestó en la vida cotidiana de las ciudades, en el uso del tiempo libre y en las costumbres (Subercaseaux, Historia de las ideas 104). La elite deseaba convertir a Chile en una nación moderna y mostrar al mundo 
este estatus $^{5}$. La práctica de la ciencia, así como la generación de conocimiento científico, otorgaban un medio para conseguir lo anterior (Silva, 2008), situación que podría explicar el alto interés de la sociedad y de la prensa por asuntos científicos. Este ambiente social de deseos de pujanza no era exclusivo de Chile, como ya decíamos, también se vivían contextos similares en otras repúblicas latinoamericanas o en zonas europeas periféricas desde el punto de vista del desarrollo científico, y en específico de la astronomía, como en España (Ruiz-Castell, "El cometa..." 170-171).

A este ambiente deben agregarse distintos hitos propios del devenir astronómico en nuestro país, tales como la llegada de una expedición de astrónomos norteamericanos, liderados por William W. Campbell, que instaló el Observatorio Mills en el Cerro San Cristóbal para obtener las velocidades radiales de las estrellas brillantes; así como el traslado del Observatorio Astronómico Nacional (OAN) desde la comunda de Quinta Normal a la de Lo Espejo. Este último acontecimiento estuvo plenamente ligado a intereses políticos acordes al discurso modernizador que defendía el presidente Pedro Montt, quien financiaría con recursos estatales la construcción del complejo astronómico más moderno de Sudamérica en Lo Espejo. No obstante, tras el inesperado fallecimiento del presidente en 1910, este proyecto nunca llegó a concretarse plenamente, hecho que deprimió sustancialmente a Federico Ristenpart, director del OAN, quien además fue denunciado en esos años por supuestas conductas irregulares en su administración, lo que concluyó con el suicidio de este astrónomo en 1913. La sufrida labor de este alemán en nuestro país, así como las infructuosas jefaturas de Alberto Obrecht antes y después del periodo de dirección de Ristenpart, hablarían por sí solas de las contradicciones del discurso modernizador asociado al desarrollo de las ciencias.

A pesar de estas noticias deplorables acerca del desarrollo de la astronomía en Chile a principios del siglo XX, la sociedad en esos años se mostraba sumamente interesada en esta ciencia, como puede verificarse en la prensa del periodo. La notificación de futuros fenómenos celestes, así como la explicación de estos, o bien, el seguimiento de alguna controversia científica o el llamado a la calma 
por parte de un astrónomo después de un sismo fueron, entre otras, las temáticas que la prensa cubrió en sus páginas durante la época (Valderrama, "La catástrofe" 178; Ramírez, "Expertos y profanos..." s/p). Como ha señalado Nieto-Galán, gracias al desarrollo y expansión de la impresión, la divulgación científica se convirtió en un negocio ("La ciencia en la esfera..." 66), de tal modo que el atractivo que despertaban en las audiencias las temáticas científicas fue aprovechado por la industria periodística y editorial. Esto último tuvo repercusiones en la manera de escribir y transmitir el conocimiento científico, ya que el lenguaje tuvo que volverse asequible al común de las personas, y la información tuvo que organizarse en formatos atractivos para audiencias de diferentes clases sociales y condiciones culturales (67).

La ciencia y la prensa, en consecuencia, se vieron sumamente relacionadas en este contexto, presentando interesantes vínculos en los llamados magazines. En ellos, como ya decíamos, gracias a su esencia miscelánea, hay amplia cabida para el diálogo entre disciplinas diversas, lo que facilita la convivencia, por ejemplo, entre el saber astronómico y el literario (Ramírez, "Expertos y profanos...” s/p).

Agustín Edwards Mac-Clure, cabeza de editorial Zig-Zag y fundador de la mayoría de los magazines que estudiamos, además de empresario periodístico, era un renombrado político y economista. Fue miembro del Partido Nacional liberal de centro-derecha, que en sus inicios en 1857 defendió la tolerancia religiosa y la secularización del Estado, y que hacia fines del siglo XIX y principios del XX representaba especialmente los intereses bancarios y económicos, que era el rubro al que se dedicaba la familia Edwards. El presidente de la República Pedro Montt también compartía esta inclinación política, por lo que durante sus años de gobierno (1906 a 1910) este partido fue denominado "monttvarismo" Edwards Mac-Clure imprimió en sus proyectos periodísticos esa idea librepensadora en un contexto donde, como ya señalamos, el desarrollo de las ciencias era visto como un puntapié para alcanzar la anhelada imagen de nación moderna (Alvarado 123).

Estas revistas, además, no se limitaron solo a transmitir conocimiento científico, sino que mucho más allá de eso, visibilizaron y cuestionaron las contradicciones entre el discurso modernizador y las características culturales de nuestra sociedad. Así, por ejemplo, Alberto Edwards,

Conjunción de los apellidos del entonces presidente Montt y del Ministro Antonio Varas. 
primo de Agustín Edwards, miembro del mismo partido político y uno de los redactores más importante de las revistas de la editorial Zig-Zag —especialmente de Pacífico Magazine—, se lamentaba de que nuestra raza, de herencia española, fuese buena para observar, pero no para imaginar: "Maravilloso cuando observa y critica, [pero] comienza a balbucear cuando propone algo... Ni en España ni aun en Chile hay discursos magistrales sino de oposición" (Edwards, "La felicidad en la..." 433). Sus palabras hacen eco del sinsabor que producía el choque entre el idealizado progreso que conseguirían los adelantos científicos y tecnológicos, y la falta de iniciativa y facultades para utilizar estos en pos de crear novedosas y efectivas soluciones a los problemas de nuestra sociedad. Así, continúa Alberto Edwards, "el sitio de la imaginación que tenemos vacío, lo ocupa la ilusión... Tras de esta imposible es que no venga el desengaño..." (434).

Este tipo de pensamiento como el que plantea Edwards, atraviesa la manera en que circula el conocimiento astronómico en los magazines estudiados, puesto que en sus páginas no solo se transmiten contenidos de esta disciplina, sino que además los cuestionan, los resignifican y los utilizan para otros propósitos. La astronomía en estas revistas puede percibirse inserta en avisos publicitarios, en caricaturas políticas y, sobre todo, en obras literarias, especialmente en narrativa de ciencia ficción, aunque también es posible encontrarla en poemas. El conocimiento astronómico se utilizaba a menudo para referirse a otros asuntos, tales como la política y la publicidad, desde una manera crítica y humorística, dando cuenta de un tratamiento del contenido científico para aludir, por ejemplo, a las incongruencias de discursos entre los adelantos científicos y la situación social, política y cultural del país. Esta utilización del saber astronómico para otros fines, a su vez, revela que los llamados "públicos de la ciencia" son agentes activos capaces de atribuir nuevos significados al conocimiento científico y, por ende, de generar nuevos contenidos en ese campo.

El fin o destrucción de nuestro planeta provocado por fenómenos celestes, se convirtió en este contexto en una temática con intereses múltiples. Por un lado, la industria editorial y periodística aprovecharía el sensacionalismo y atracción de las audiencias que provocaban las predicciones apocalípticas de algunos astrónomos; por otro, los mismos astrónomos utilizarían las controversias en torno a las supuestas predicciones para legitimarse como autoridades o expertos científicos 
frente a la sociedad ${ }^{7}$; finalmente, los escritores tomarían estas teorías predictivas como motivos de inspiración de sus obras literarias, porque al hacerlo contaban con altas probabilidades de que se las publicaran. De esta manera, las predicciones en torno a la destrucción de nuestro planeta a partir de daños provocados por los astros será una temática ampliamente explotada por la prensa de masas de fines del siglo XIX y principios del XX y, en consecuencia, eclipses solares, el paso de cometas, la cercanía de asteroides a la Tierra y los terremotos ${ }^{8}$, activarían la generación y circulación de conocimiento astronómico en estas revistas.

A continuación, se analizan las relaciones entre los distintos focos de interés presentes en los magazines de editorial Zig-Zag - directores, editores, redactores de temas científicos, escritores literarios o lectores-, respecto de la significación de asuntos astronómicos vinculados con la destrucción del planeta, concentrándonos especialmente en la lectura de textos de ciencia ficción aparecidos en estas revistas a principios del siglo XX.

\section{La destrucción del planeta y la narrativa de ciencia ficción en los magazines}

El alto interés de la sociedad por asuntos astronómicos en este periodo puede constatarse, entre otras fuentes, en la constante publicación de ciencia ficción en las páginas de estas revistas, lo que se complementa con la circulación de libros del género inspiradas en fenómenos celestes, como las novelas de Julio Verne, que fueron traducidas en nuestro país en esas décadas (Subercaseaux, La historia del libro en Chile 72).

Las controversias entre científicos siempre han sido una instancia que ofrece oportunidades a estos para legitimar su condición de expertos en alguna materia. Este fenómeno ha sido ampliamente estudiado por los siguientes autores: Lankford (1981), Dascal (1998), Gross (1998), Freudenthal (1998), Machamer et al. (2000), Ruiz-Castell (2011).

8 Lorena Valderrama (2016; 2017) ha realizado amplios estudios acerca de la sismología en Chile en este periodo, y entre las teorías que circulan para explicar los terremotos se encuentran algunas vinculadas con los efectos producidos por los astros. Así mismo, a falta de instituciones dedicadas a la sismología en nuestro país, los pocos sismógrafos se encontraban dentro de los observatorios astronómicos, lo que obligaba a los astrónomos a pronunciarse frente a la sociedad cada vez que se producía un sismo considerable. 
El origen de la ciencia ficción generalmente se atribuye a la novela Frankenstein (1818) de Mary Shelley ${ }^{9}$. Desde entonces, este género cautivó a un gran número de lectores. La ciencia ficción consiste en "writing a story that preserves something like the effects of a disturbing dream, while grounding those effects in plots that not depend on supernatural events" (Alkon 5). En ese sentido, la provocación de efectos que no dependen de elementos sobrenaturales puede ser el factor que incita el acercamiento de este género hacia lo científico, puesto que si bien no se puede echar mano a lo sobrenatural para ir más allá de lo humanamente posible, sí se puede recurrir potencialmente a ello mediante la fusión de la imaginación y la experimentación científica. Las obras de ciencia ficción, por consiguiente, se nutren del contenido científico y no funcionan sin este, por lo que, a su propósito conscientemente literario, puede añadirse el rol divulgativo. Así, por ejemplo, en las novelas de Julio Verne se proporcionan al lector, en clave literaria, elementos sólidos de cultura científica (Nieto-Galán, "La ciencia en la esfera...” 73).

Se debe tener en cuenta que a fines del siglo XIX y principios del XX no existían límites claros entre la pura ficción literaria, la ciencia ficción y la divulgación científica, por lo que era común que las obras literarias inspiraran la escritura de textos de divulgación (como fue el caso de los textos de Louis Figuier), o que científicos crearan obras de ciencia ficción para reforzar su programa de divulgación (como Camille Flammarion), favoreciendo que la escritura de los difusores de la ciencia se mantuvieran entre la novela, el texto académico y la divulgación (Nieto-Galan, "La ciencia en la esfera..." 74-75). En ese sentido, la ciencia ficción no necesariamente se presenta en formato de novela o cuento, sino que también habría que considerar textos de divulgación atravesados profundamente por la ciencia ficción, de tal modo que se han borrado sus límites genéricos.

En la ciencia ficción la temática de la destrucción de la Tierra es reiterativa, de tal modo que se podría hablar de este leitmotiv como un subgénero dedicado a lo apocalíptico (Novell 263). La destrucción del planeta causada específicamente por fenómenos astronómicos, es igualmente repetitiva en la ciencia ficción, lo que puede constatarse

\footnotetext{
Como señala Noemí Novell, el origen de este género es debatible. Existen tres tendencias respecto de cuándo y quién habría iniciado la ciencia ficción y, entre ellas se encuentra la atribuida a Mary Shelley. Independientemente de las distintas teorías, Novell explica que el punto de inicio de cualquier género es impreciso, en cuanto que es una entidad de fronteras difícilmente estáticas (21-28).
} 
a través de los magazines chilenos del periodo. Esto último no es particular del contexto chileno, sino que su práctica proviene de Europa y Norteamérica, y se presenta en términos parecidos en otros países de Latinoamérica.

Un caso interesante que responde a esa dificultad de delimitar el género es un texto de Camille Flammarion publicado en el $\mathrm{n}^{\circ} 12 \mathrm{de}$ Revista Zig-Zag el 7 de mayo de 1905. Flammarion (1842-1925) fue un reconocido astrónomo francés y divulgador de la astronomía, cuyas obras tuvieron alcance en gran parte del orbe. Una de las maneras que encontró este científico para popularizar la astronomía fue la escritura de obras de ciencia ficción ${ }^{10}$ y la narración de textos de divulgación que generalmente incluían proyecciones de situaciones ficticias explicadas a través de contenidos científicos. Este comportamiento puede verse claramente en su texto aparecido en Revista Zig-Zag, titulado "El fin del mundo". Allí el autor fundió datos y cálculos científicos con párrafos colmados de situaciones ficticias hipotéticas, en las que un fenómeno celeste podría provocar caos y destrucción en nuestro planeta:

La velocidad de un cometa en el espacio dirigiéndose hacia la órbita de la Tierra, es igual a la de nuestro planeta multiplicada por la raíz cuadrada de 2, es decir, 106.70o kilómetros por hora (término medio) multiplicado por 1.414. Si ese astro cabelludo llegase de frente sobre nosotros, el choque representaría pues una velocidad de $\mathbf{2 5 7 . 0 0 0 ~ k i l o ́ m e t r o s ~ p o r ~ h o r a . ~ S u p o n g a m o s ~}$ que un cometa de las mismas dimensiones que el de 1811 llegue precisamente sobre nosotros en su viaje circular alrededor del Sol. (...) La travesía de la cabeza cometaria de 1.800.00o kilómetros de diámetro duraría 25.000 segundos, o sea 417 minutos o seis horas 57 minutos. El incendio se declararía muy luego; nuestra atmósfera se inflamaría como una ponchera. No se producirían cientos sino miles de grados, el oxígeno del aire le costaría muy poco para alimentar las llamas, y el hidrógeno del mar se desprendería muy fácilmente" (Flammarion 3).

El texto continúa "ficcionalizando" mediante cálculos de contenido científico. Los subtítulos que le siguen son: "La asfixia o la locura suprema espera a la humanidad”, “Todos los continentes serán un día ahogados 
como en un naufragio", "Muerte universal por medio del frío”, "La Tierra se convertirá fatalmente en un oscuro desierto de hielo", lo que da cuenta de la pronunciada retórica apocalíptica. El texto, en consecuencia, es una interesante mezcla entre ciencia ficción y difusión científica, en el que el autor utilizó temáticas que despertaban alto interés en la audiencia, como era el caso de la toxicidad del aire que podría ocasionar la cercanía de un cometa: "Una mezcla cometaria de óxido de carbono de nuestra atmósfera traería la supresión rápida de todas las respiraciones por medio del envenenamiento de la sangre" (Flammarion 6), explica el astrónomo, acompañándose el texto con ilustraciones que amplifican la significación devastadora de sus palabras.

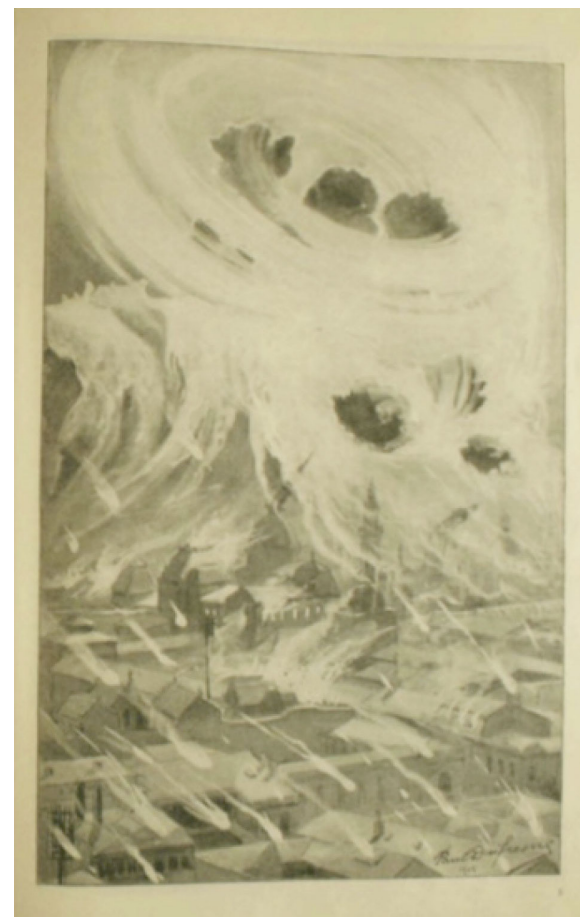

La posible toxicidad de la cola de los cometas está relacionada con el análisis espectroscópico que permite identificar los elementos que componen los cuerpos celestes, estudio que comienza a aplicarse con los inicios de la astrofísica en el siglo XIX ${ }^{11}$. Morehouse (1876-1941) descubrió en 1908 un nuevo cometa que según el análisis espectroscópico 
de su contorno portaba gases altamente tóxicos para los seres humanos (Ruiz-Castell, “El cometa...” 174). Este tema cobró mucha atención durante la primera década de 1900 por el inminente paso del cometa Halley en 1910. A pesar de que la mayoría de los astrónomos descartaba peligros ocasionados por la interacción de este astro con nuestro planeta, "los divulgadores científicos habrían de explotar la incertidumbre de un posible paso de la Tierra por la cola del cometa" (174). Entre esos divulgadores se encontraba Flammarion.

Camille Flammarion fue uno de los autores de ciencia ficción mayormente publicado en los magazines chilenos del periodo, y uno de los que mayor número de respuestas generó después de la aparición de cada uno de sus textos, aunque no fue el único. Simon Newcomb (1835-1909), astrónomo y escritor norteamericano y canadiense, también publicó ciencia ficción en estas revistas. En Pacífico Magazine, aparece su cuento "El fin del mundo" en noviembre de 1914, que trata sobre un profesor de ciencias que sabe de la presencia de un astro que va en dirección al sol, y que lo hará brillar y calentar más que nunca, acabando con la vida en la Tierra. En sus páginas se lee lo siguiente justo después del momento en que el astro choca con el Sol:

Sus rayos azotaron el continente como un torrente colérico. A medida en que avanzaba del Atlántico al Pacífico, todas las cosas combustibles que alcanzaba a tocar se incendiaron. Las piedras se deshicieron con el calor, torres y campanarios cayeron estrepitosamente como si las sacudiera un terremoto. Los hombres procuraron buscar refugio en los subterráneos o debajo de alguna cobertura que pudiera protegerlos del calor atroz. Viejos y jóvenes, ricos y pobres, hombres y mujeres, se estrechaban unos a otros en la confusión de los desesperados (Newcomb, "El fin del mundo" 565).

La frase final de este novelista revela una concepción religiosa del fin de los tiempos asociada al Juicio Final, en el que supuestamente todos los seres humanos serían juzgados por igual, sin importar la condición que tuvieron en la Tierra. El texto también fue acompañado de ilustraciones que potenciaban el tono apocalíptico de la narración. 


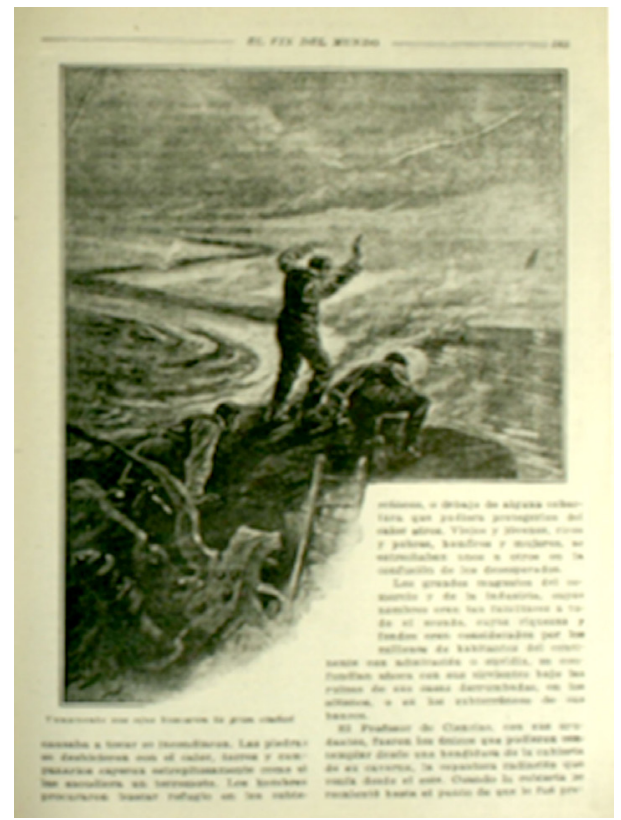

Dentro de las reacciones a nivel global y local frente a este tipo de textos, había expertos que rechazaban las especulaciones llamando a la calma a la población, así como profanos de la ciencia que optaban por seguir los consejos de estos últimos o por alarmarse y temer que la predicción fuese cierta. Las páginas de los magazines chilenos nos permiten hacer un seguimiento de las respuestas locales (de expertos, escritores y lectores en Chile) respecto de la circulación global de este tipo de temáticas.

Así, por ejemplo, en 1907 encontramos un artículo en el n 236 de Sucesos titulado "Un gran cometa que vuelve" que hace alusión a que el Halley se aproximaría a la Tierra en el año 1910. En este texto, el autor (redactor local que no firma) enfatiza en que el terror hacia este tipo de fenómenos celestes es un sentimiento retrógrado: "Las visitas del cometa de Halley producían en los siglos de superstición e ignorancia verdadero terror, engendrado por el extraño aspecto en el que se presentaba aquel" (s/p). Luego explica mediante conceptos científicos otra visión respecto del fin del mundo, señalando que la venida del cometa Halley permitirá a los científicos observar la composición del astro y constatar lo que han venido proponiendo diversos científicos acerca de la constante descomposición de partículas que sufren los cuerpos celestes, lo que podría llevar a calcular el fin del universo: 
Todo esto tiene un gran significado respecto del destino final del Universo. Primitivamente los astrónomos creían ver en los fenómenos celestes simples ejemplos de evolución avanzando siempre. Pero ahora preséntase al estudio de los hombres de ciencia extraños fenómenos que parecen demostrar una marcha inversa. En efecto, los cometas se descomponen en partículas y van siendo dispersados poco a poco (s/p).

El tono de este texto, al contrario del de Flammarion y Newcomb, incita a la calma, y si bien alude al tema del fin del mundo, apunta a explicar desde una perspectiva netamente científica, que el cometa Halley traería consigo no destrucción, sino una oportunidad para estudiar su composición y el futuro del universo.

Sucesos publicó otro artículo titulado “Cosas del cielo" en su ${ }^{\circ}$ 393, el 17 de marzo de 1910, dos meses antes del paso del Halley. En este, el autor local afirma que todas las elucubraciones respecto de si la cola del cometa alcanzaría nuestra atmósfera e intoxicaría a la población humana no tienen ningún sentido: “Es, pues, prematuro todo cuanto se diga sobre el particular y a nada conduce sino a causar alarmas infundadas e inútiles hablares sobre el envenenamiento en masa del género humano y del fin del mundo" (s/p). Cuando el redactor explica que es prematuro hablar de la toxicidad de la cola del cometa, revela que conoce el análisis espectroscópico a través del cual los astrofísicos habían comenzado a estudiar la composición del Halley, arrojando presencia de cianógeno ${ }^{12}$ en el núcleo del astro. Justamente la posibilidad de que este gas mortal se esparciera por su cola, y de que esta última alcanzara nuestro planeta, era lo que había encendido la alarma en la población. Este temor que el autor califica como infundado, por consiguiente, tiene un asidero científico - el análisis espectroscópico-, por lo que se vuelve interesante corroborar que los primeros exámenes del cometa realizado por expertos habían circulado rápidamente entre la audiencia lega. El recelo frente al paso de este cometa se relaciona directamente con el descubrimiento de Morehouse que ya hemos mencionado, que consistió en el análisis espectroscópico del área brillante del contorno de otro cometa más lejano a la Tierra

12 El cloruro de cianógeno es un compuesto químico extremadamente tóxico. Causa un daño inmediato al entrar en contacto con los ojos o con las vías respiratorias. Los síntomas producto de su exposición pueden ser desde pérdida del conocimiento a parálisis y muerte. 
en 1908, y que demostró contener varios gases, entre ellos cianógeno (Ruiz-Castell 174). Aunque la mayoría de los astrónomos afirmaba que no había peligro inminente asociado a este fenómeno - ya que en el peor de los casos apenas se evidenciaría una inofensiva lluvia de meteoros ${ }^{13}$ - , los divulgadores y la prensa explotaron esta temática, aprovechando el clima de incertidumbre en torno a la aproximación e intensidad de la cola del cometa.

El 23 de marzo de 1910, es decir, seis días después del artículo de Sucesos, el magazine Corre-Vuela en su $\mathrm{n}^{\circ} 117$, publicó un artículo titulado "Otra vez el fin del mundo", haciendo alusión a la recurrencia con la que circulaban este tipo de predicciones. Esta vez el autor se dirigió directamente a Camille Flammarion, acusándolo de atemorizar irresponsablemente a la población: "Camilo Flammarion, el gran astrónomo francés, de vez en cuando se complace en intimidar a los incautos con profecías basadas en hechos científicos, pero que son, sin embargo, algo exageradas" (s/p). El tono de este autor local para referirse a la situación predictiva es de cansancio, asegurando una vez más a la audiencia de que el paso del cometa Halley en mayo no provocaría ningún inconveniente para la humanidad.

Sin embargo, a pesar de los esfuerzos de estos textos (a menudo redactados o apoyados por astrónomos locales) por calmar a los lectores y desmentir los pronósticos de exterminio que podría generar el paso cercano de un cometa, e incluso después de verificarse que el Halley no produjo ninguna catástrofe, la sugestión y la incertidumbre sobre posibles daños ocasionados por fenómenos celestes permaneció en la sociedad. Así nos lo demuestra la publicación del cuento de Simon Newcomb sobre el que ya nos referimos, en noviembre de 1914 de Pacífico Magazine, y la sección de Preguntas y Respuestas de Revista Zig-Zag en el mismo mes en su $n^{\circ} 507$. En esta parte del magazine se publicaban inquietudes redactadas por lectores dirigidas a los editores, como la siguiente de uno que firma como José P.: "Muy señor mío: Fuera de lo que nos enseña la religión, ¿qué se sabe de cierto acerca del fin del mundo?” (s/p). Esto da cuenta de que entre los legos se mantiene el interés en el tema. La respuesta que da el editor se fundamenta en palabras de Henri Poincaré (1854-1912), matemático, físico, científico teórico y filósofo de la ciencia, francés, y dice así:

13 Para profundizar en los posibles efectos del paso del Cometa Halley, véase Campbell (1909). 
Cuanto al fin lento y natural del mundo vivo, opinan los sabios que habrá de suceder sea por falta de agua, o por el contrario por exceso de este elemento, o por extinción del sol, es decir, que la vida perecerá de frío. Luego sería explicar cómo puede cualquiera de estas tres hipótesis realizarse trayendo consigo la muerte de todo lo que vive en el mundo. Cuanto a la ruina o fin del mundo todo o sea del Universo, Henry Poincaré la admite y explica de esta manera (s/p).

El fin del mundo ocasionado por los astros durante este periodo fue tan comentado, que los caricaturistas y escritores lo llegaron a tratar de manera burlesca. A través del humor, utilizaron la temática para enlazarla con otros asuntos de actualidad, tales como la crítica política y social. Esto se puede observar desde los primeros años del siglo XX, como es el caso del texto "La fin du monde" publicado en el n 211 de la Revista Zig-Zag y firmado por las iniciales PSCH. El escrito data de septiembre de 1906, un mes después del terremoto de Valparaíso:

La culpa de estas catástrofes [refiriéndose al terremoto] no la tiene nadie más que nosotros mismos. Hay que distinguir: una cosa es la lava y otra cosa es la lava y después la plancha... que estamos haciendo. Porque aquello de: "Señores diputados: ¡Estamos sobre un volcán!”, ya no asusta a nadie. La mayor parte de los volcanes sirve para que los vayan a ver unos cuantos turistas. ¡Nada más! (...) El planeta demasiado aguante ha tenido soportándonos durante tantos miles de años (...) permitiéndonos (...) a todos que ganaran lo que se les antojase (...) ¡Hay otros astros y quiere que nos mudemos a cualquiera de ellos! (s/p).

Este fragmento, mediante un tono burlesco, funde contenidos astronómicos y político-sociales de manera audaz. Rechaza la teoría sísmica de que los terremotos fuesen ocasionados por fenómenos volcánicos, diciendo: “Tenemos que desechar por absurda la teoría plutónica de que la Tierra es algo así como tetera puesta al fuego; lo mismo pudiera ser cafetera y así no habría té-rremotos sino caferremotos" (s/p). En el primer fragmento puede verse cómo el autor vincula dicha teoría con álgidas situaciones políticas, aludiendo a estas últimas como "volcanes". Antes de estas dos citas, el texto se inicia con un párrafo donde el autor llama la atención de los supuestos "expertos" en materia sísmica en el país, que como ya hemos adelantado, en esta época 
correspondían a la figura de los astrónomos. En esta parte el redactor critica primero a Curtis, director del Observatorio Mills en este periodo: "Aunque diga Mr. Curtis que él se explica lo que ha pasado [el fenómeno sísmico], yo también me lo explico, con la diferencia de que para eso él cobra sueldo" (s/p). Luego arremete contra Obrecht, director del Observatorio Astronómico Nacional en esos años: “Otro a quien habrá que decirle ¡explíquese usted! es a Obrecht, este buen señor que negó la existencia de un cometa en vez pasada ${ }^{14}$, se ha quedado ahora mudo, no sabemos si de terror o de espanto" (s/p).

Se debe advertir que la mayoría de los autores de ciencia ficción encontrados en páginas de revistas chilenas eran extranjeros. No obstante, deben considerarse tan interesantes como sus colaboraciones - algunas de ellas exclusivamente escritas para nuestros lectores locales $-{ }^{15}$, las reacciones o respuestas locales dentro del país provocadas por este tipo de obras. En ese sentido, podemos decir que la ciencia ficción de autoría extranjera publicada en los magazines estudiados produce una instancia de generación activa de conocimiento astronómico local constatable en las páginas de estas revistas. Ese conocimiento provendría de las múltiples respuestas de los públicos locales de la ciencia, ya sea de expertos o profanos, siendo la temática de predicciones del fin del mundo por causas astronómicas un fenómeno que hubo de intensificar este proceso. La literatura de ciencia ficción, entonces, funciona dentro de estos magazines como un motor de activación para la producción de conocimiento astronómico, y a su vez, como hemos podido observar, la literatura opera en sí misma como un dispositivo de divulgación de contenidos científicos.

\section{Conclusión}

A través de los apartados anteriores se ha podido identificar uno de los modos en que circuló el saber astronómico en revistas magazinescas

\footnotetext{
$14 \quad$ Puede referirse a una conferencia dada por Obrecht en la Sociedad Científica en 1899 en la que se refirió a las predicciones infundadas de Falb, quien pronosticaba un posible choque del cometa Biela y la Tierra con resultados desastrosos en noviembre de ese año. La conferencia de Obrecht fue publicada en Revista de Chile el 1 de noviembre de 1899.

15 Flammarion enviaba correspondencia especialmente dirigida a La Lira chilena. Esto puede constatarse en el no 14 de dicha revista, de agosto de 1898, donde aparece la primera colaboración que responde a este convenio, titulada "En el mundo de los astros: un nuevo cometa". El cuento "El fin del mundo" de Newcomb, por otra parte, fue exclusivamente traducido para Pacífico Magazine, como puede constatarse en su no 20 , pp. 557.
} 
chilenas a principios del siglo XX. En este ejercicio se ha evidenciado, a su vez, la importancia del género literario y, en específico, de la ciencia ficción, en la movilidad y popularización de este conocimiento científico.

Gracias a su carácter misceláneo, los magazines fundados en Chile durante las primeras décadas del siglo XX fueron medios que promovieron el diálogo entre múltiples disciplinas y variadas temáticas, característica que puede corroborarse a través del análisis de las predicciones de la destrucción del mundo asociadas a fenómenos astronómicos en las revistas de editorial Zig-Zag. En ellas, como ha podido verse, las teorías científicas en torno a la toxicidad de la cola de los cometas incentivaron la creación literaria y, a su vez, la ficción basada en este motivo permeó las páginas de textos de divulgación científica.

El análisis de este asunto en los magazines también permite corroborar la manera en que circulaba el conocimiento astronómico entre expertos e inexpertos, dando cuenta, al contrario de lo que podría pensarse, que los primeros no siempre llamaron a la calma y a no creer en predicciones relacionadas con el paso de un cometa, sino que incluso varios de esos expertos, como Flammarion y Newcomb, alimentaron esas teorías con su escritura divulgativa y literaria en contextos globales. Los profanos y audiencias locales, por otra parte, reaccionaban manifestándose a favor o en contra de dichas teorías, interrogando y pidiendo explicaciones a los expertos, o bien, como hemos explicado anteriormente, utilizando el saber astronómico para aplicarlo a otras materias, tales como la política, la crítica social y la publicidad, otorgando nuevos significados a teorías y conocimientos científicos.

En consecuencia, los magazines estudiados funcionaron como dispositivos que favorecieron la transdisciplinariedad - como la que se ha podido observar- entre discursos literarios y teorías científicas. De este modo, estos proyectos editoriales además de aprovechar para fines comerciales el sensacionalismo que despertaban las teorías predictivas, se convirtieron en espacios de circulación del saber científico y en agentes activos de generación de conocimiento astronómico, en cuanto que motivaron la discusión científica a partir de diversas interpretaciones de fenómenos celestes. 


\section{Referencias bibliográficas}

Alkon, Paul. Science Fiction Before 190o: Imagination, Discovers, Technology. New York \& London, Routledge, 2002.

Alvarado, Marina. Revistas culturales y literarias chilenas de 1900 a 1920: legitimadoras del campo literario nacional. Santiago de Chile, Editorial Cuarto Propio, 2016.

Anónimo. “Un gran cometa que vuelve”. Revista Sucesos, no. 236 (1907): s/p. . “Cosas del cielo”. Revista Sucesos, no. 393 (1910): s/p.

. “Otra vez el fin del mundo”. Revista Corre-Vuela, no. 117, 1910, s/p.

Broks, Peter. "Science, Media and Culture: British Magazines, 1890-1914". Sci, no. 2, 1993, pp. 123-139.

Campbell, William Wallace. “The Return of Halley's Comet”. Publications of the Astronomical Society of the Pacific, no. 21, 1909, pp. 188-195.

Cantor, Geoffrey, et al. Science in the nineteenth century periodical: Reading the magazine of nature. Cambridge, Cambridge University Press, 2004.

Castany, Bernat. "El motivo del fin del mundo en la literatura hispanoamericana". Tropelias. Revista de Teoría de la Literatura y Literatura Comparada, no. 25, 2016, pp. 183-191.

Dascal, Marcelo. "The Study of Controversies and the Theory and History of Science". Science in Context, vol. 11, no. 2, 1998, pp. 147-154.

Edwards, Alberto. "La felicidad en la vida modesta”. Pacífico Magazine, no. 25,1915 , pp. 432-434.

Flammarion, Camille. “El fin del mundo”. Revista Zig-Zag, no. 12, 1905, pp. 3-8.

Freudenthal, Gideon. "Controversy". Science in Context, vol. 11, no. 2, 1998, pp. 155-160.

Gingerich, Owen. "Astrophysics and twentieth-century astronomy to 1950". The General History of Astronomy. London, Cambridge University Press, 1984.

Gross, Alan. "Do the Disputes over Priority Tell Us Anything about Science?". Science in Context, vol. 11, no. 2, 1998, pp. 161-179.

Lankford, John. "Amateurs versus Professionals: The Controversy over Telescope Size in Late Victorian Science”. Isis, vol. 72, no. 261, 1981, pp. 11-28. 
Latorre, José Antonio. "La poesía de la muerte y del fin del mundo". Textualia. Revista de Letras y Humanidades, no. 3, 2015, pp. 2-15. Recuperado de https://www.academia.edu/31633940/ LA POES\%C3\%8DA DE LA MUERTE Y DEL FIN DEL MUNDO

Latour, Bruno. La ciencia en acción. Barcelona, Editorial Labor, 1992.

Machamer, Peter, et al. Scientific Controversies. Philosophical and Historical Perspectives. New York, Oxford University, 2000.

Newcomb, Simon. "El fin del mundo". Pacífico Magazine, no. 20, 1914, pp. 557-569.

Nieto-Galán, Agustí. Los públicos de la ciencia. Expertos y profanos a través de la historia. Madrid, Marcial Pons, 2011.

"La ciencia en la esfera pública del siglo XIX: géneros, discursos y apropiaciones". Cultura Escrita \& Sociedad, no. 10, 2010, pp. 53-80.

Novell, Noemí. Literatura y cine de ciencia ficción. Perspectivas teóricas. Tesis doctoral Universidad Autónoma de Barcelona, 2008. Impreso.

Obrecht, Alberto. "El sistema planetario y el choque de los cometas. Conferencia dada en la Sociedad Científica de Chile, por A. Obrecht". Revista de Chile, vol. 3, 1899, pp. 266-269.

Ossandón, Carlos y Eduardo Santa Cruz. El estallido de las formas. Chile en los albores de la cultura de masas. Santiago de Chile, LOM Editores, 2005.

P., José. "Preguntas y respuestas”. Revista Zig-Zag, no. 507, 1914, s/p.

PSCH. "El fin del mundo". Revista Zig-Zag, no. 211, 1906, s/p.

Ramírez, Verónica. "Expertos y profanos: Circulación del saber astronómico en magazines chilenos (1900-1920)". Revista de Humanidades. Aceptado.

Ruiz-Castell. "El cometa de Halley y la imagen pública de la astronomía en la prensa diaria española de principios del siglo XX". Dynamis, no. 33, 2013, pp. 169-193.

Ruiz-Castell, Pedro. "Priority Claims and Public Disputes in Astronomy: E. M. Antoniadi, J. Comas i Solà and the Search of Authority and Social Prestige in the Early Twentieth Century". British Journal for the History of Science, no. 44, 2011, pp. 509-531. 
Sanhueza, Carlos. La movilidad del saber científico en América Latina: objetos, prácticas e instituciones (siglos XVIII al XX). Santiago de Chile, Universitaria, 2018.

Secord, James. "Knowledge in Transit”. Isis, no. 95, 2004, pp. 654-672.

Shapin, Steven \& Simon Schaffer. Leviathan and the Air-Pump: Hobbes, Boyle, and the Experimental Life. Princeton, Princeton University Press, 1985.

Silva, Bárbara. Identidad y nación entre dos siglos. Patria Vieja, Centenario y Bicentenario. Santiago de Chile, LOM Editores, 2008.

Subercaseaux, Benjamín. Historia de las ideas y de la cultura en Chile. Santiago de Chile, Universitaria, 2004.

La historia del libro en Chile (Alma y Cuerpo). Santiago de Chile, LOM Editores, 2000.

Valderrama, Lorena. "La catástrofe anunciada: terremotos y predicciones en la prensa diaria chilena (1906-1912)”. Ciencia y espectáculo. Circulación de saberes científicos en América Latina, siglos XIX y XX. Eds. María José Correa, Andrea Kottow y Silvana Vetö. Santiago Chile, Ocho Libros, 2016.

- Observando la catástrofe, terremotos y conocimiento sísmico en Chile (1868-1912). Tesis doctoral Universidad de Valencia, 2017. Impreso. 